\title{
Gender-Specific Outcomes after Percutaneous Left Atrial Appendage Closure - A Nationwide Readmission Database Analysis
}

Neel Patel ${ }^{1}$, Sagar Ranka ${ }^{1}$, Adrija Hajra ${ }^{2}$, Dhrubajyoti Bandyopadhyay $^{3}$, Birendra Amgai ${ }^{4}$, Sandipan Chakraborty ${ }^{5}$, Mazin Khalid ${ }^{6}$, Amandeep Goyal ${ }^{1}$, Tarun Dalia ${ }^{1}$, Madhu Reddy $^{1}$, and Jacob Shani ${ }^{6}$

${ }^{1}$ University of Kansas Medical Center

${ }^{2}$ Jacobi Medical Center

${ }^{3}$ Westchester Medical Center Health Network

${ }^{4}$ Geisinger Community Medical Center

${ }^{5}$ Miami Valley Hospital

${ }^{6}$ Maimonides Medical Center

November 17, 2021

\begin{abstract}
Background: Thromboembolism-associated stroke is the most feared complication of Atrial fibrillation (AF). Percutaneous left atrial appendage closure (pLAAC) is indicated for stroke prevention in patients with AF who can't tolerate long-term anticoagulation. We aim to study gender differences in peri-procedural and readmissions outcomes in pLAAC patients. Methods: Using the national readmission database from January 2016 to December 2018, AF patients undergoing the pLAAC procedure were identified. We used multivariate logistic regression analyses and time-to-event Cox regression analyses to conduct the study. Propensity matching with the Greedy method was done for the accuracy of results. Result: 28,819 patients were included in our study. Among them 11,946 (41.5\%) were women and 16,873 (58.6\%) were men. The mean age of overall population was $76.1 \pm 8.5$ years, with women 1 year older than men. The overall rate of complications was higher in women $(8.6 \%$ vs $6.6 \%$, $\mathrm{P}<0.001$ ), primarily driven by bleeding-related complications i.e., Major bleed (OR: $1.3295 \% \mathrm{CI}: 1.03-1.69$, p=0.029), blood transfusion (OR: 1.45, 95\% CI: 1.06-1.97, p=0.019) and cardiac tamponade (OR: 1.80, 95\% CI: 1.13-2.89, p=0.014). Women had two times higher peri-procedural ischemic stroke. There was no difference in peri-procedural mortality. Women remained at $20 \%$ and $13 \%$ higher risk for readmission at 30 days and 6 months of discharge. Conclusion: Women had higher peri-procedural complications and were at higher risk of readmissions at 30 days and six months. However, there was no difference in mortality during the index hospitalization. Further studies are necessary to determine causality.
\end{abstract}

Gender-Specific Outcomes after Percutaneous Left Atrial Appendage Closure - A Nationwide Readmission Database Analysis

\section{Authors:}

1. Neel Patel, MD University of Kansas Medical Center, Kansas City, KS, USA Email: neelpatel.ny@gmail.com

2. Sagar Ranka, MD University of Kansas Medical Center, Kansas City, KS, USA Email: sranka@kumc.edu

3. Adrija Hajra, MBBS Jacobi Medical Center, New-York, NY, USA Email: adrija847@gmail.com

4. Dhrubajyoti Bandyopadhyay, MD Westchester Medical Center, New York, NY, USA Email: drdhrubajyoti87@gmail.com 
5. Birendra Amgai, MD Geisinger Community Medical Center, Scranton, PA Email: biren.amgai08@gmail.com

6. Sandipan Chakraborty, MD Miami Valley Hospital, Dayton, OH Email: schakraborty591@gmail.com

7. Mazin Khalid, MD Maimonides Medical Center, New York, NY Email: dr.mazinkhalid@gmail.com

8. Amandeep Goyal, MD University of Kansas Medical Center, Kansas City, KS, USA Email: docaman2k1@live.com

9. Tarun Dalia, MD University of Kansas Medical Center, Kansas City, KS, USA Email: tdalia2@kumc.edu

10. Y.Madhu Reddy, MD University of Kansas Medical Center, Kansas City, KS, USA Email: yreddy@kumc.edu

11. Jacob Shani, MD Maimonides Medical Center, New York, NY Email: jshani@maimonidesmed.org

\section{Corresponding author:}

Neel Patel, MD

Kansas University Medical Center, Kansas City, KS, USA

Email: neelpatel.ny@gmail.com

$1^{\text {st }}, 2^{\text {nd }}$, and $10^{\text {th }}$ author had equal contribution

\section{Conflict of interest: Authors declare no conflict of interest}

\section{Funding: (None)}

\section{Institutional Review Board approval: Not Applicable}

Gender-Specific Outcomes after Percutaneous Left Atrial Appendage Closure - A Nationwide Readmission Database Analysis

\section{Abstract: \\ Background:}

Thromboembolism-associated stroke is the most feared complication of Atrial fibrillation (AF). Percutaneous left atrial appendage closure (pLAAC) is indicated for stroke prevention in patients with AF who can't tolerate long-term anticoagulation. We aim to study gender differences in peri-procedural and readmissions outcomes in pLAAC patients.

Methods:

Using the national readmission database from January 2016 to December 2018, AF patients undergoing the pLAAC procedure were identified. We used multivariate logistic regression analyses and time-to-event Cox regression analyses to conduct the study. Propensity matching with the Greedy method was done for the accuracy of results.

Result:

28,819 patients were included in our study. Among them 11,946 (41.5\%) were women and 16,873 (58.6\%) were men. The mean age of overall population was $76.1 \pm 8.5$ years, with women $\sim 1$ year older than men. The overall rate of complications was higher in women ( $8.6 \%$ vs $6.6 \%, \mathrm{P}<0.001)$, primarily driven by bleedingrelated complications i.e., Major bleed (OR: 1.32 95\% CI: 1.03-1.69, p=0.029), blood transfusion (OR: 1.45, 95\% CI: 1.06-1.97, $\mathrm{p}=0.019$ ) and cardiac tamponade (OR: 1.80, 95\% CI: 1.13-2.89, $\mathrm{p}=0.014$ ). Women had two times higher peri-procedural ischemic stroke. There was no difference in peri-procedural mortality. Women remained at $20 \%$ and $13 \%$ higher risk for readmission at 30 days and 6 months of discharge.

Conclusion: 
Women had higher peri-procedural complications and were at higher risk of readmissions at 30 days and six months. However, there was no difference in mortality during the index hospitalization. Further studies are necessary to determine causality.

Key words: Left atrial appendage, readmissions, stroke, bleeding, hospitalization

Abbreviation:

$\mathrm{AF}$ - Atrial Fibrillation

pLAAC - percutaneous Left Atrial Appendage Closure

NRD - National Readmission Database

ESRD - End Stage Renal Disease

$95 \%$ CI - 95\% confidence interval

OR - Odds Ratio

HR - Hazard Ratio

\section{Introduction:}

Atrial fibrillation (AF) is the most common arrhythmia encountered in clinical practice. The lifetime risk of developing AF in a 55-year-old person is as high as 40\%.[1] Thromboembolism-associated stroke is the most feared complication of AF.[2] In AF patients, the risk of stroke increases by 4-fold in men and 5.7-fold in women. [3] Percutaneous left atrial appendage closure (pLAAC) is a novel technique for stroke prevention in patients with AF who are not suitable candidates for long-term anticoagulation.[4]

Multiple prior studies have reported significant gender differences in risk of AF and antecedent stroke risk, with women at a considerably higher risk of stroke and hospital admissions. [5, 6] Initial European experience with pLAAC showed women were at higher risk of cardiac tamponade/pericardial effusion.[7] The pivotal trials leading to the approval of pLAAC were not powered to compare gender-specific differences rigorously. $[8,9]$ The National Cardiovascular LAAO Registry reported increased rates of major complications in women at the time of implantation; however, short-term risk thereafter remains unknown.[10] Therefore, we aim to study gender differences in peri-procedural and readmissions outcomes in patients undergoing pLAAC.

\section{Method:}

\section{Data Source:}

We utilized the national readmission database (NRD) from January 2016 to December 2018 to conduct this study.[11] NRD is the largest publicly available all-payer inpatient health care readmission database in the US, containing data from 17 million discharges (unweighted) each year. When weighted, it provides roughly 36 million discharges, accounting for $58 \%$ of the total US population and $57 \%$ of all US hospitalizations. The NRD is part of a family of databases and software tools developed through a Federal-State-Industry partnership for the Healthcare Cost and Utilization Project (HCUP). It includes a full calendar year of data with diagnosis and procedure codes using the International Classification of Diseases, Tenth Revision, Clinical Modification Diagnostic, and Procedure Coding System (ICD-10-CM and PCS). Due to deidentified nature of this database, this study did not require Institutional Review Board approval.

\section{The study protocol and patient selection:}

Between January 2016 to December 2018, we identified patients admitted with AF using appropriate ICD-10CM codes. (Supp. file) AF patients who had pLAAC were identified using the ICD-10-PCS code. Readmission was determined following the methodology described under the HCUP. We documented only the first readmission if the patient was admitted multiple times. Trauma-related readmissions were excluded before the final analysis. The details of the inclusion process are presented in Figure 1. We evaluated gender-specific index hospitalization complications and mortality. For the 30-day readmission analyses, we excluded patients 
admitted in the December months of the study period. For six-month readmission analyses, we excluded patients from July to December months of the study period as patients can't be tracked into the following year. Additionally, we assessed overall and gender-specific predictors of readmissions and the top 10 causes of readmissions in men and women.

Statistical Method:

All statistical analyses were performed using STATA (Version 16).[12] Weighting of patient-level observations was implemented to obtain national estimates. Propensity matching was done by the Greedy method. Multivariate regression analysis models were built by including all confounders that were significantly associated with the outcome on univariable analysis with a cutoff P-value of 0.2 . Variables that were deemed important determinants of the outcomes based on literature were forced into the models. Binary outcomes such as index hospitalization mortality and complications were compared by logistic regression. For readmission comparison, we used the time to event cox regression analysis. Demographic variables were compared using the Student t-test. All p-values were two-sided, with 0.05 as a threshold for statistical significance.

\section{Results:}

Baseline Characteristics:

A total of 28,819 patients met our inclusion criteria and were included in our study. Among them 11,946 $(41.5 \%)$ were women and $16,873(58.6 \%)$ were men. The mean age of overall population was $76.1 \pm 8.5$ years, with women slightly older than men (women:76.6 \pm 8.2 yrs vs. men:75.8 \pm 8.7 years; $\mathrm{p}<0.001$ ). Most patients were covered by Medicare, followed by private payer coverage. Women had a higher prevalence of anemia $(\mathrm{p}<0.001)$, chronic lung disease $(\mathrm{p}<0.001)$, history of stroke $(\mathrm{p}=0.005)$, obesity $(\mathrm{p}=0.005)$, and valvular heart disease $(\mathrm{p}=0.033)$. Whereas men had a high proportion of Diabetes $(\mathrm{p}<0.001)$, coronary artery disease $(\mathrm{p}<0.001)$, peripheral arterial disease $(\mathrm{p}<0.001)$, and its related complications. (Table 1$)$.

\section{Index hospitalization complications and mortality:}

The overall rate of complications was higher in women than men ( $8.6 \%$ vs. $6.6 \%, \mathrm{P}<0.001)$. After adjusting of baseline variables using propensity-matched multivariate logistic regression analysis, we found complications were primarily driven by bleeding-related complications, i.e., Major bleed (OR: 1.32 95\% CI: 1.03-1.69, $\mathrm{p}=0.029$ ), blood transfusion (OR: $1.45,95 \% \mathrm{CI}: 1.06-1.97, \mathrm{p}=0.019$ ) and cardiac tamponade (OR: 1.80 , 95\% CI: $1.13-2.89, \mathrm{p}=0.014)$. Women were more likely to have a peri-procedural ischemic stroke (OR: 2.03, $95 \%$ CI:1.01-4.10, $\mathrm{p}=0.049)$. On propensity-matched multivariate logistic regression analysis, we found no statistically significant difference in peri-procedural mortality between women and men (OR: 1.88, 95\% CI: 0.74-4.79, $\mathrm{p}=0.185$ ). (Table-2) Mean length of stay (LOS) was longer in women (women:1.4 \pm 2.4 days vs. men:1.3 \pm 1.8 days, $\mathrm{p}<0.001)$. Women were more likely to be discharged to skilled nursing facilities $(\mathrm{SNF})$ (women:3.4\% vs. men:1.9\%, $\mathrm{p}<0.001$ ).

\section{Readmission outcomes:}

A total of 2,496 (8.7\%) patients were readmitted within 30-days of discharge. Higher proportion of women were readmitted compared to men $[1,139(9.6 \%)$ vs $1,357(8 \%) ; \mathrm{p}<0.001]$. The time to readmission was also shorter in women (women:12.2 \pm 9 days vs. men:13.3 \pm 9 days, $\mathrm{p}=0.023$ ). On propensity-matched multivariate time-to-event cox regression analysis, at 30 days, women were more likely to readmit (HR: 1.20, 95\% CI:1.04-1.38, $\mathrm{p}=0.015$ ), which was also true at 180 days (HR: 1.13, 95\% CI:1.01-1.27, $\mathrm{p}=0.047$ ). (Table3) Subgroup analysis for the readmission due to hemorrhagic causes and thromboembolic causes showed no difference. Condition such as high Charlson category $(\mathrm{p}<0.001)$, End-stage renal disease (ESRD) $(\mathrm{p}<0.001)$, anemia $(p<0.001)$, pulmonary hypertension $(\mathrm{p}=0.001)$, and chronic lung diseases $(\mathrm{p}=0.022$ were associated with more readmissions. Additionally, elective procedures $(\mathrm{p}=0.020)$ were associated with lower readmissions (supplementary table).

The common causes of readmissions were congestive heart failure, gastrointestinal bleeding, sepsis, and acute kidney injury. Women had more readmissions due to AF. (Supplementary table) 


\section{Discussions:}

In our study of 28000 pLAAC procedures, we found significant gender-specific differences in outcomes during index hospitalization as well as during subsequent readmissions. Specific findings of our study include A) Women had higher overall complications primarily driven by bleeding-related complications. i.e., 1.3fold increased risk of major bleeding, 1.8-fold higher risk for cardiac tamponade, and 2-fold augmented risk of ischemic stroke. B) There was no gender-specific difference in peri-procedural mortality, c) Women remained at $20 \%$ and $13 \%$ higher risk for readmission at 30 and 180-days, respectively, d) High Charlson category, ESRD, anemia, pulmonary hypertension, and chronic lung diseasease were significant predictors of subsequent readmissions. Elective procedures were associated with lower readmissions.

A study published by Freeman and his colleagues reported pLAAC device utilization is gradually increasing since the approval of the WATCHMAN device in 2015[13]. Additionally, the reported adverse event rates were much lower than the pivotal PROTECT-AF and PREVAIL trial. In the PROTECT-AF trial, subgroup analysis showed a reduction of primary efficacy endpoint (composite of stroke, cardiovascular or unexplained death, and systemic embolization) in men, but there was no difference in women[9]. The PREVAIL trial, which evaluated device efficacy and adverse events, did not report gender-specific analysis[8]. Our comparative analysis reported higher peri-procedural complications rates in women, which was primarily driven by hemorrhagic complications. The study results align with the recently published National Cardiovascular Data Registry (NCDR), Left atrial appendage occlusion (LAAO) registry-based study by Daren et al.[10] Determining the causality of this difference becomes more pressing as pLAAC devices are now being compared to novel oral anticoagulants as a second-line treatment for AF patients.[14]

Though our study can not determine the causality, few possible explanations can be relatable. A quantitative study based on the Mayo Clinic tissue registry showed the anatomical difference in LAA between men and women.[15] In men, LAAs are wider and longer, while in women, they are shorter, have more volume, and have a high orifice location. $[16,17]$ This difference, along with older age, might be associated with a frail LAA wall, contributing to higher pericardial complications. Bleeding during index hospitalization might be related to smaller and shorter common femoral artery size in women [18], leading to difficult access, and a high prevalence of anemia may exacerbate the situation. Resuarringly, though complications were higher in women, there was no difference in mortality.

Our study showed women are at $20 \%$ and $13 \%$ higher risk for readmission at 30 days and 6-months. Late clinical referral, advanced age, multiple co-morbidity, higher bleeding risk, and more peri-procedural complications might be associated with prolonged LOS and lead to more readmissions in women. Since atrial fibrillation is associated with higher thromboembolic and bleeding complications in women, we carried out subgroup analysis to determine a cause-specific gender-based difference of readmission[3]. It showed no difference between men and women in both thromboembolic and bleeding-related readmissions, suggesting equal efficacy in both groups.

Careful pre-procedural anatomical evaluation of LAA anatomy through advanced imaging and intervening electively (vs. urgent), when feasible, may help to reduce peri-procedural complications and in-term readmissions. Appropriately managing the risk factors such as peri-procedural pericardial complications can also play an essential role in avoiding readmissions. Pre-procedural management of patients' past conditions such as anemia, renal disease, valvular disease, and chronic lung diseases through a systemic approach and incorporating guidelines in routine practice might reduce the readmission burden in this cohort. Postprocedural close follow-up and interval monitoring for GI bleed and renal function should be encouraged.

\section{Limitations:}

Being an administrative database, NRD is subjected to coding errors. pLAAC is an operator-dependent procedure. NRD does not have information regarding the type and size of the device used, echocardiographic findings, pre, and post-procedure imaging results. Patients readmitted to another state cannot be traced in the NRD, and mortality data cannot be calculated outside the hospital after discharge. Despite all the limitations, our analysis results are in accordance with the data reported in previous studies. $[9,10,19]$ 
Because it uses an enormous sample size, it can also provide insights into uncommon risk factors, a limitation of a single-center cohort study.

\section{Conclusion:}

This study is the most extensive study reporting 30 days and 6-months readmission rates based on gender to the best of our knowledge. Women have more peri-procedural complications primarily driven by hemorrhagic complications. However, there was no difference in mortality. Identifying and managing the risk factors of readmission can reduce the healthcare burden. Further risk stratification and mitigation strategies need to be explored.

\section{References:}

1. Staerk, L., et al., Lifetime risk of atrial fibrillation according to optimal, borderline, or elevated levels of risk factors: cohort study based on longitudinal data from the Framingham Heart Study. BMJ, 2018. 361 : p. k1453.

2. Wolf, P.A., R.D. Abbott, and W.B. Kannel, Atrial fibrillation as an independent risk factor for stroke: the Framingham Study. Stroke, 1991. 22 (8): p. 983-8.

3. Michaud, G.F. and W.G. Stevenson, Atrial Fibrillation. New England Journal of Medicine, 2021. 384 (4): p. 353-361.

4. January, C.T., et al., 2019 AHA/ACC/HRS Focused Update of the 2014AHA/ACC/HRS Guideline for the Management of Patients With Atrial Fibrillation: A Report of the American College of Cardiology/American Heart Association Task Force on Clinical Practice Guidelines and the Heart Rhythm Society in Collaboration With the Society of Thoracic Surgeons. Circulation, 2019. 140 (2): p. e125-e151.

5. Westerman, S. and N. Wenger, Gender Differences in Atrial Fibrillation: A Review of Epidemiology, Management, and Outcomes. Curr Cardiol Rev, 2019. 15 (2): p. 136-144.

6. Emdin, C.A., et al., Atrial fibrillation as risk factor for cardiovascular disease and death in women compared with men: systematic review and meta-analysis of cohort studies. BMJ (Clinical research ed.), 2016.532 : p. h7013-h7013.

7. Schmidt, B., et al., Incidence of pericardial effusion after left atrial appendage closure: The impact of underlying heart rhythm-Data from the EWOLUTION study. J Cardiovasc Electrophysiol, 2018.29 (7): p. 973-978.

8. Holmes, D.R., Jr., et al., Prospective randomized evaluation of the Watchman Left Atrial Appendage Closure device in patients with atrial fibrillation versus long-term warfarin therapy: the PREVAIL trial. J Am Coll Cardiol, 2014. 64 (1): p. 1-12.

9. Holmes, D.R., et al., Percutaneous closure of the left atrial appendage versus warfarin therapy for prevention of stroke in patients with atrial fibrillation: a randomised non-inferiority trial. Lancet, 2009. 374 (9689): p. 534-42.

10. Darden, D., et al., Sex Differences in Procedural Outcomes Among Patients Undergoing Left Atrial Appendage Occlusion: Insights From the NCDR LAAO Registry. JAMA Cardiol, 2021.

11. (HCUP), H.C.a.U.P. NRD Overview . 07/15/2021]; Available from: https://www.hcupus.ahrq.gov/nrdoverview.jsp.

12. StataCorp, Stata Statistical Software: Release 16 . 2019, College Station, TX: StataCorp LLC.

13. Freeman, J.V., et al., The NCDR Left Atrial Appendage Occlusion Registry. J Am Coll Cardiol, 2020. 75 (13): p. 1503-1518.

14. Wintgens, L.I.S., et al., The WATCHMAN left atrial appendage closure device for patients with atrial fibrillation: current status and future perspectives. Expert Review of Medical Devices, 2020.17 (7): p. 615-626. 
15. Veinot, J.P., et al., Anatomy of the normal left atrial appendage: a quantitative study of age-related changes in 500 autopsy hearts: implications for echocardiographic examination. Circulation, 1997. 96 (9): p. 3112-3115.

16. Boucebci, S., et al., Assessment of normal left atrial appendage anatomy and function over gender and ages by dynamic cardiac CT. Eur Radiol, 2016. 26 (5): p. 1512-20.

17. Elzeneini, M., A. Elshazly, and A.E.M. Nayel, The left atrial appendage morphology and gender differences by multi-detector computed tomography in an Egyptian population. Egypt Heart J, 2020.72 (1): p. 38.

18. Sandgren, T., et al., The diameter of the common femoral artery in healthy human: influence of sex, age, and body size. Journal of vascular surgery, 1999. 29 (3): p. 503-510.

19. Osman, M., et al., Sex-stratified analysis of the safety of percutaneous left atrial appendage occlusion. Catheter Cardiovasc Interv, 2021. 97 (5): p. 885-892.

\section{Hosted file}

Tables and figures.docx available at https://authorea.com/users/446669/articles/545853gender-specific-outcomes-after-percutaneous-left-atrial-appendage-closure-a-nationwidereadmission-database-analysis 